\title{
Erratum: Magnetoresistance of a three-dimensional Dirac gas [Phys. Rev. B 98, 195420 (2018)]
}

\author{
Viktor Könye and Masao Ogata
}

(Received 11 June 2019; published 20 June 2019)

DOI: 10.1103/PhysRevB.99.249904

The following equations should be changed to

$$
\begin{gathered}
a:=\frac{\ell_{B}}{\sqrt{2}}\left(\pi_{x}-i \pi_{y}\right), \quad a^{\dagger}:=\frac{\ell_{B}}{\sqrt{2}}\left(\pi_{x}+i \pi_{y}\right) \\
G_{b a}=\frac{1}{V^{2}} \sum_{\boldsymbol{k}, \boldsymbol{k}^{\prime}} \boldsymbol{\phi}_{b}^{\dagger}(\boldsymbol{k}) \boldsymbol{G}_{\boldsymbol{k} \boldsymbol{k}^{\prime}} \boldsymbol{\phi}_{a}\left(\boldsymbol{k}^{\prime}\right) \\
\Sigma_{b a}\left(i \omega_{m}\right)=\frac{1}{V^{2}} \sum_{\boldsymbol{k}, \boldsymbol{k}^{\prime}} \boldsymbol{\phi}_{b}^{\dagger}(\boldsymbol{k}) \boldsymbol{\Sigma}_{\boldsymbol{k} \boldsymbol{k}^{\prime}}\left(i \omega_{m}\right) \boldsymbol{\phi}_{a}\left(\boldsymbol{k}^{\prime}\right) \\
-\pi \delta\left(E_{a}-\mathcal{M}\right)=-\pi \frac{\mathcal{M}}{\sqrt{\mathcal{M}^{2}-\mathcal{D}^{2}}} \delta\left(\mathcal{P}_{z} \pm \sqrt{\mathcal{M}^{2}-\mathcal{D}^{2}}\right)
\end{gathered}
$$

The first three equations have minor misspellings that do not affect the results after them. The last one changes the result for $\sigma_{x x}$ for the $\Delta \neq 0$ case. The interpretation after Eq. (55) should be changed to:

"In the case of $\mathcal{D}=0$, this has no magnetic-field dependence and, thus, $C_{a b} \propto \ell_{B}^{q+1}$ leading to $\sigma_{x x} \propto \ell_{B}^{q}$. But, in the case of $\mathcal{D} \neq 0$, Eq. (55) is proportional to $B$ since the density of states becomes larger getting closer to the bottom of the Landau level. But the scattering rate decays at a larger rate in the massive case, and thus, in the end, $\sigma_{x x} \propto \ell_{B}^{q}$, the same way as the massless case. Since the $n=0$ case decays the least rapidly, it will be dominant at high magnetic fields so the overall magnetic-field dependence of the conductivity becomes $\sigma_{x x} \propto B^{-1}$ for both $\Delta=0$ and $\Delta \neq 0$."

Figures 11-13 have to be replaced also because of a numerical error. The difference is only in the figures with finite mass terms and only in the high-field region. We can see that the difference between the massive and the massless cases is not as big as was suggested. The different behavior is at intermediate magnetic fields, and the two cases are quantitatively different at high fields.
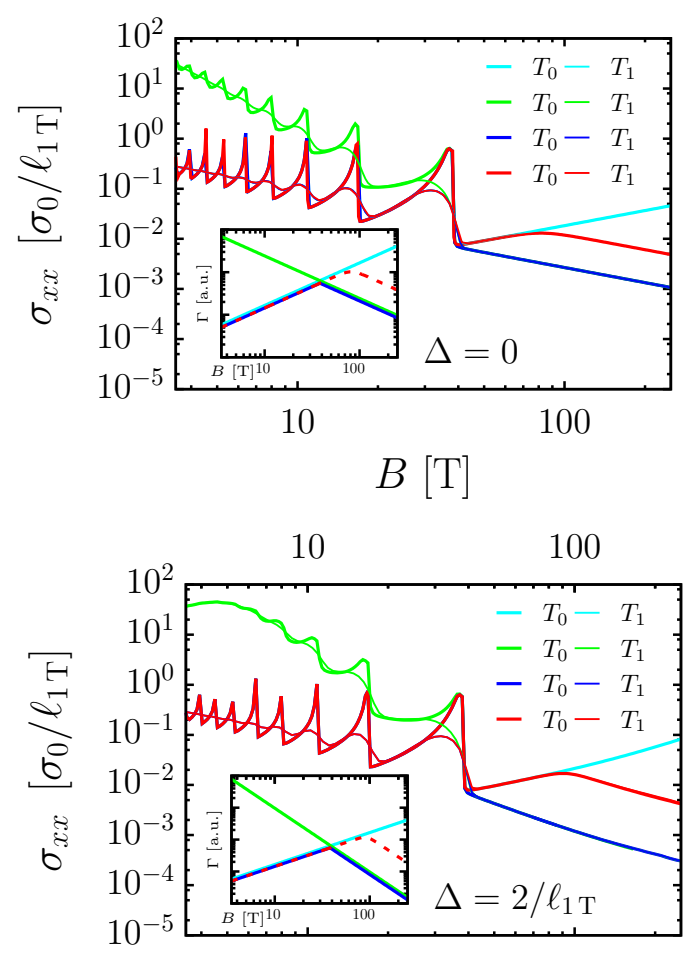

FIG. 11. Transverse diagonal conductivity $\sigma_{x x}$ calculated from Eq. (52) as a function of magnetic field at different temperatures. $\Delta=0$ (top plot) and $\Delta=2 / \ell_{1 \mathrm{~T}}$ (bottom plot). The scattering rate is chosen phenomenologically based on the numerical results in Fig. 8. The inset shows the scattering rates used (no Landau-level dependence is assumed). $T_{0}=0, T_{1}=50 \mathrm{~K}$, the density of charge carriers is $n_{e}=10^{18} \mathrm{~cm}^{-3}$, and $\sigma_{0} / \ell_{1 \mathrm{~T}} \approx 15 \Omega^{-1} \mathrm{~cm}^{-1}$. 

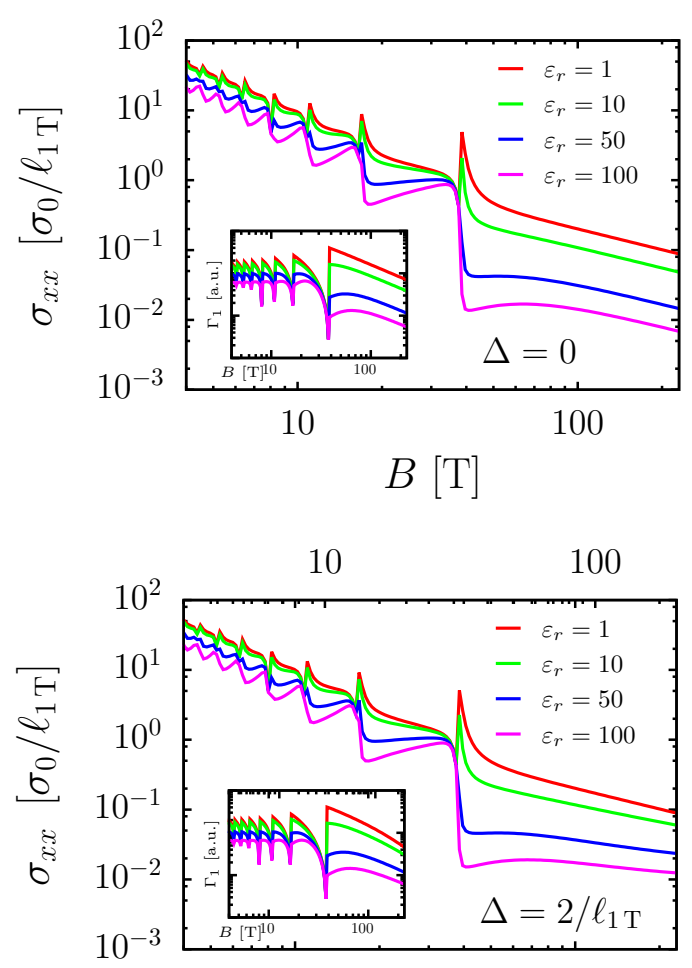

FIG. 12. Transverse diagonal conductivity $\sigma_{x x}$ calculated from Eq. (52) as a function of magnetic field at zero temperature. $\Delta=$ 0 (top plot) and $\Delta=2 / \ell_{1 \mathrm{~T}}$ (bottom plot). The scattering rate is calculated using Eq. (46a) using screening wave numbers calculated through Eq. (41). The inset shows the scattering rates used $(n=1$ Landau level). The density of charge carriers is $n_{e}=10^{18} \mathrm{~cm}^{-3}$, and $\sigma_{0} / \ell_{1 \mathrm{~T}} \approx 15 \Omega^{-1} \mathrm{~cm}^{-1}$.
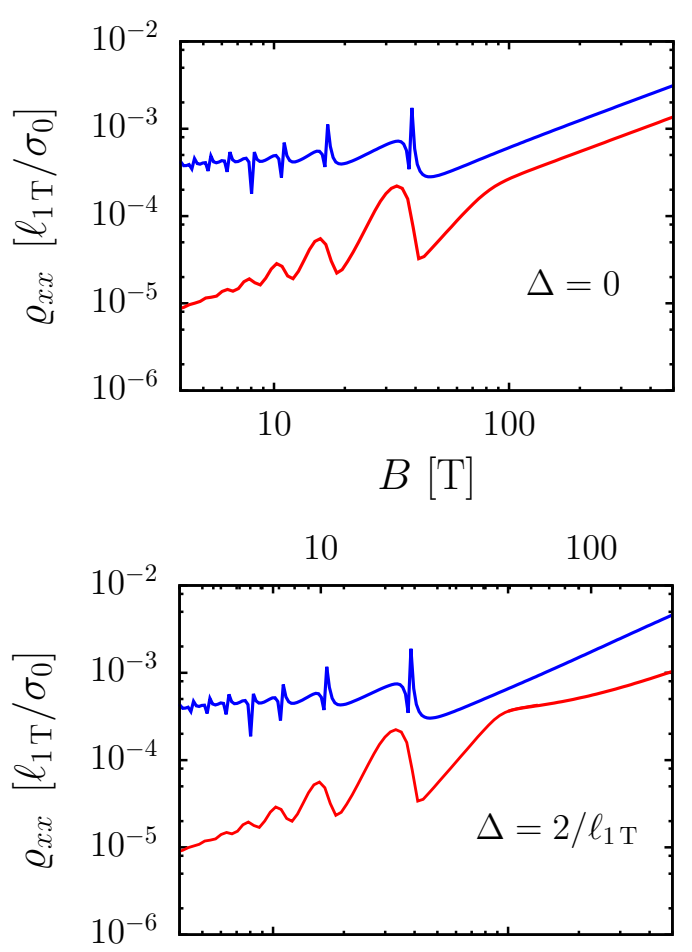

FIG. 13. Magnetoresistance $\varrho_{x x}$ calculated from Eq. (56) as a function of magnetic field. $\Delta=0$ (top panel) and $\Delta=2 / \ell_{1 \mathrm{~T}}$ (bottom panel). The resistivity calculated from the phenomenological result (red line) and the resistivity calculated microscopically using the first Born approximation with $\varepsilon=1$ (blue). The density of charge carriers is $n_{e}=10^{18} \mathrm{~cm}^{-3}$, and $\sigma_{0} / \ell_{1 \mathrm{~T}} \approx 15 \Omega^{-1} \mathrm{~cm}^{-1}$. 